Family Profile No. 5, 2021

\title{
Median Age at Last Birth
}

Authors: Lisa Carlson \& Karen Benjamin Guzzo

Trends and differentials in the age at first birth are well-documented (FP-20-06). Given shifts and variation in completed family size (FP-20-04), it is also important to look at the age of last birth - that is, when do women stop having children - which has received very little attention. This profile investigates the median age at last birth among women at the end of their childbearing years, at 45-49 years old. Using the 2015-2019 cycles of the National Survey of Growth, this profile investigates the median age at last birth for mothers aged 45-49 by race/ethnicity, completed education, parity, and age at first birth.

$>$ Among all mothers aged 45-49, the median age at last birth was 31 years of age.

\section{Median Age at Last Birth by Race and Ethnicity}

The median age at last birth varied slightly between women across racial/ethnic groups.

- Hispanic women and women of 'Other' race/ethnicity had the oldest median ages at last birth at 32 years of age.

- The youngest median age at last birth was among Black women at 30 years of age.
Figure 1. Median Age at Last Birth by Race/Ethnicity

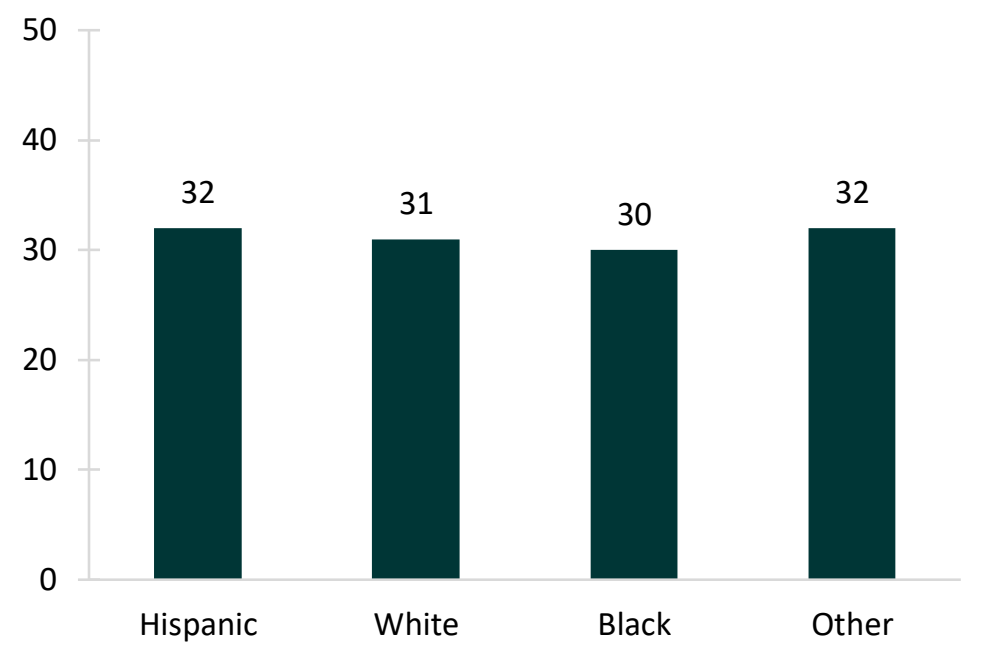

Source: NCFMR analyses of female data files from NSFG cycles 201517 and 2017-2019

\section{Median Age at Last Birth by Completed Educational Attainment}

Figure 2. Median Age at Last Birth by Educational Attainment

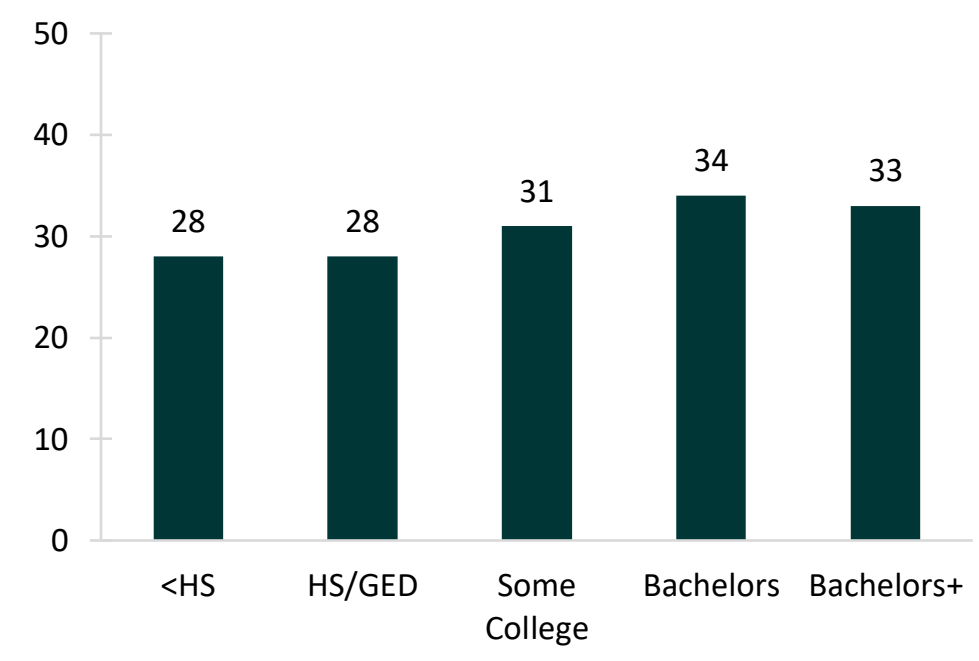

Source: NCFMR analyses of female data files from NSFG cycles 201517 and 2017-2019
- The median age at last birth generally increased with increased levels of educational attainment.

- Women with a high school education or less had the youngest median age at last birth (28 years).

$0 \quad$ The oldest median age at last birth was among women with a bachelor's degree (34 years).

- For women with more than a high school degree, the median age at last birth was older than 30 years of age. 


\section{Median Age at Last Birth by Parity}

- For parity, the median age at last birth increased with higher parities.

- The median age at last birth for women with at least four children was five years older than the median age at last birth among women with one child (34 compared to 29 years of age).
Figure 3. Median Age at Last Birth by Parity

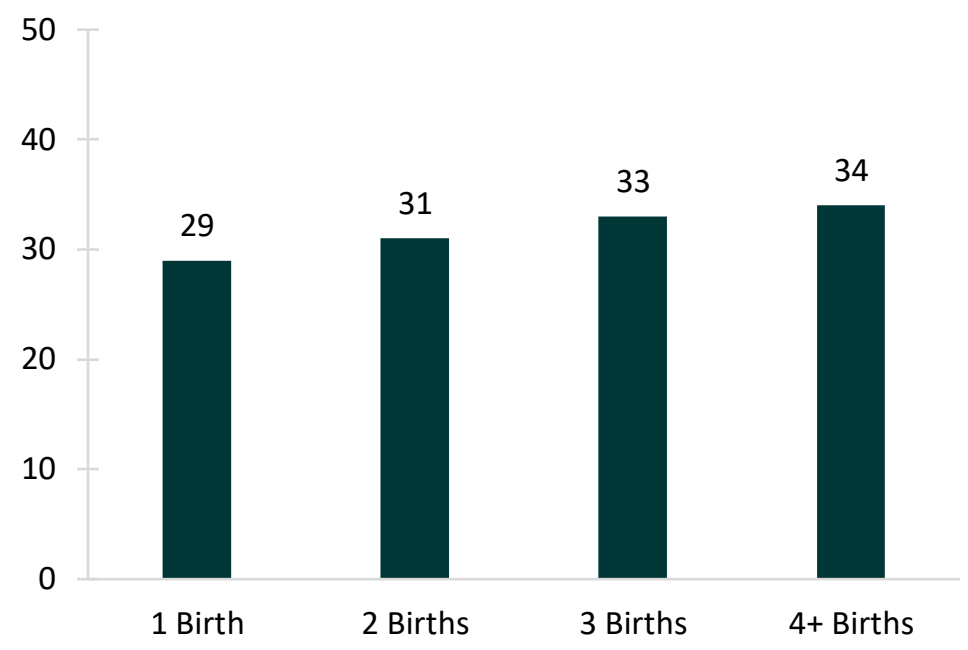

Source: NCFMR analyses of female data files from NSFG cycles 201517 and 2017-2019

\section{Median Age at Last Birth by Age at First Birth}

Figure 4. Median Age at Last Birth by Age at First Birth

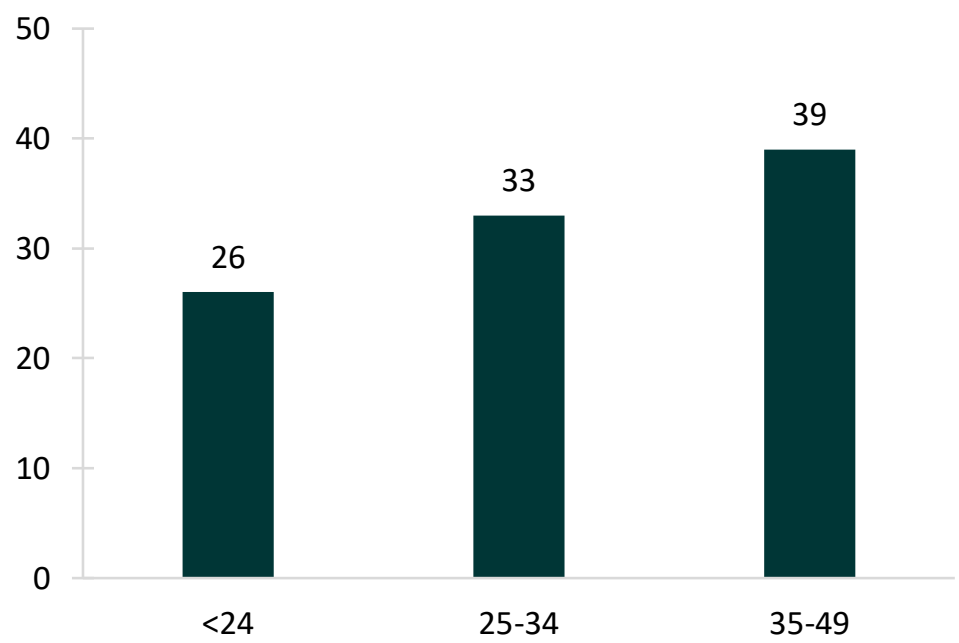

- Source: NCFMR analyses of female data files from NSFG cycles 2015-17 and 2017-2019
The later women began having children, the later they finished having children.

- The median age at last birth was more than a decade later for women who had a first birth at age 35 or older compared to women who had a first birth before age 24 ( 39 and 26 years of age, respectively).

\section{Data Sources:}

National Center for Health Statistics (NCHS). 2015-17 and 2017-2019 National Survey of Family Growth Public-Use Data and Documentation. Hyattsville, MD: CDC National Center for Health Statistics. https://www.cdc.gov/nchs/nsfg/index.htm

\section{References:}

Guzzo, K. B. \& Schweizer, V. (2020). Number of children to women aged 40-44, 1980-2018. Family Profiles, FP-20-04. National Center for Family \& Marriage Research. https://doi.org/10.25035/ncfmr/fp-20-04

Schweizer, V. \& Guzzo, K. B. (2020). Age at first birth among mothers 40-44, 1990 \& 2018. Family Profiles, FP-20-06. Bowling Green, OH: National Center for Family \& Marriage Research. https://doi.org/10.25035/ncfmr/fp-20-06

\section{Suggested Citation:}

Carlson, L. \& Guzzo, K. B. (2021). Median age at last birth. Family Profiles, FP-21-05. Bowling Green, OH: National Center for Family \& Marriage Research. https://doi.org/10.25035/ncfmr/fp-21-05 